\title{
Template pour une carrière académique en médecine de famille
}

\author{
Ryan Tandjung ${ }^{a}$, Andreas Zeller ${ }^{b}$, Peter Jünic, Thomas Rosemann ${ }^{a}$, Thomas Bischoff ${ }^{d}$, Johanna Sommer

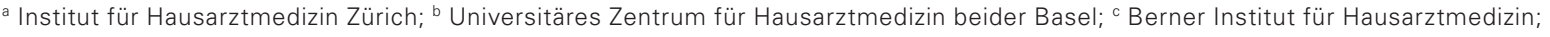 \\ ${ }^{d}$ Institut Universitaire de Médecine Génerale, Lausanne; ${ }^{e}$ Unité de Médecine de Premiers Recours, Genève
}

Les instituts universitaires de médecine de famille représentent la référence académique pour les médecins de famille (médecins internistes généralistes et pédiatres) qui s'engagent dans l'enseignement et dans la recherche. Grâce à leur infrastructure, ils assurent le soutien et la continuité nécessaires à une position forte, à la fois en clinique, en recherche et dans l'enseignement. Les instituts de médecine de famille s'illustrent par leur activité de promotion et de renforcement de l'académisation de la médecine de famille dans les domaines indissociables de la clinique, de la recherche et de l'enseignement.

\section{Activité clinique}

Les instituts ont comme tâche de garantir une activité clinique en médecine de famille de haute qualité. Dans ce but les instituts de médecine de famille élaborent des recommandations pour une médecine de famille efficiente et basée sur les évidences, correspondant à la population spécifique de médecine de premier recours. Un médecin de famille ayant une fonction académique poursuit son activité clinique afin d'assurer le lien avec le patient et avec l'essence de la profession.

\section{Recherche}

L'activité de recherche est d'une importance primordiale pour les instituts de médecine de famille. Les médecins de famille ayant une fonction académique font de la recherche à un niveau international et suscitent ainsi la reconnaissance pour leur discipline au sein de la faculté. L'objectif de la recherche en médecine de famille est l'amélioration du soin pour les patients au cabinet médical et le développement de l'enseignement. L'infra-structure assure un accès facilité aux compétences méthodologiques, par ex. en statistique ou épidémiologie. Ceci garantit la continuité des projets et permet la transmission d'une compétence méthodologique aux médecins de famille chercheurs. La recherche en médecine de famille tend vers une collaboration étroite entre les instituts de médecine de famille et les autres disciplines.

Correspondance: PD Dr Ryan Tandjung UniversitätsSpital Zürich Pestalozzistrasse 24 CH-8091 Zurich ryan.tandjung[at]usz.ch

\section{Enseignement}

L'enseignement de la médecine de famille s'appuie sur la meilleure évidence disponible, tant pour son contenu que pour sa forme didactique. Les instituts de médecine de famille s'engagent afin que la pédagogie appliquée dans les cours, séminaires et au cabinet corresponde aux critères de qualité de l'enseignement en médecine. Dans cet enseignement, une attention particulière est portée au contact avec le patient. Grâce à la recherche en pédagogie médicale, les instituts de médecine de famille stimulent le développement des compétences pédagogiques dans leur domaine. Les médecins de famille actifs dans l'enseignement devraient être intégrés dans la planification du curriculum, afin de garantir une présence adéquate de médecins de famille dans les modules tout au long du cursus.

\section{Promotion de la relève}

Les futurs médecins de famille académiques devraient être recrutés durant leur formation postgraduée en médecine interne générale (par ex. durant leurs stages dans les cliniques universitaires). Conseils et accompagnement sur le chemin vers une nomination au titre de privat-docent sont essentiels. Il est recommandé au médecin académique en médecine de famille de s'engager dans les trois domaines (clinique, recherche et enseignement), même s'il privilégie un ou l'autre domaine académique en particulier.

\section{Formation postgraduée et continue}

Les instituts de médecine de famille jouent un rôle important dans la formation postgraduée et continue. Ils participent à la coordination et s'assurent de la qualité des lieux de formation des programmes d'assistanat au cabinet et des curricula intégrés. Pour la formation continue ils publient des prises de position et des recommandations de prise en charge des patients sur la base de la littérature et de leur propre recherche. Lors des manifestations de formation continue ils s'engagent à transmettre aux médecins de famille suisses des recommandations fondées sur les preuves, des contenus de formation spécifique pour la médecine de famille de même que les résultats de l'activité de recherche en médecine de famille.

\section{Annex}

Cette prise de position est dans le contexte de Swiss Academy of Family Medicine (Safmed), la coopération des cinq instituts universitaires de médecine de famille. Un grand merci à Adrian Göldlin (Bern), Lilli Herzig (Lausanne), Dagmar Haller-Hester (Genève) et Peter Tschudi (Bale) pour leur contribution à cette position. 